\title{
Commentary: The power of indeterminacy
}

\author{
Alessandro Brunelli, MD
}

\footnotetext{
From the Department of Thoracic Surgery, St James's University Hospital, Leeds Teaching Hospital NHS Trust, Leeds, United Kingdom.

Disclosures: Author has nothing to disclose with regard to commercial support.

Received for publication Sept 15, 2019; revisions received Sept 15, 2019; accepted for publication Sept 16, 2019; available ahead of print Oct 16, 2019.

Address for reprints: Alessandro Brunelli, MD, Department of Thoracic Surgery; St James's University Hospital

Bexley Wing, Beckett St, Leeds, LS9 7TF, United Kingdom (E-mail: alexit_2000@yahoo.com).

J Thorac Cardiovasc Surg 2020;159:2041

$0022-5223 / \$ 36.00$

Copyright (c) 2019 by The American Association for Thoracic Surgery

https://doi.org/10.1016/j.jtcvs.2019.09.047
}

The study of Raman and colleagues ${ }^{1}$ in this issue of the Journal is an important study about the influence of indeterminate resection margins on long-term prognosis. Raman and colleagues ${ }^{1}$ found that patients with indeterminate margins ( $\mathrm{Rx}$ ) have a worse overall survival than do those with radical (R0) resections; they are much more like patients with microscopic residual disease (R1). In addition, Raman and colleagues ${ }^{1}$ found that adjuvant therapy was not associated with improved long-term outcome in these patients.

In the National Cancer Database, surgical margins are classified as indeterminate when they are not evaluable for technical or biologic reasons. There may be several subgroups that belong to this category, such as a disrupted specimen at the level of the tumor, a tumor breaching the visceral pleura in close proximity to adhesions, or a tumor abutting the resection margin after removal of the stapled line. Some situations are more like that of a microscopically positive resection margin; others may indicate a more aggressive biology of the tumor. Unfortunately, the nature of the database precluded a more granular analysis of the different situations, which would have added to the meaningfulness of the study.

Another major limitation of the study (again inherent in the database used for the analysis) is the lack of information about disease-free survival and recurrences. This information would have clarified the real impact of indeterminate margins on disease progression. Raman and colleagues ${ }^{1}$ tried to circumvent this limitation empirically by excluding in the first instance deaths that occurred within 90 days, assuming that those deaths were related more to treatment than to cancer recurrence. The 90-day cutoff appears arbitrary, however, and patients may continue to die for treatment-related causes even after that time. This is particularly true for high-risk patients with severe comorbidities and compromised cardiopulmonary function and for those undergoing larger resections (ie, pneumonectomy). Information regarding treatment-related versus cancer-related mortality would be important to further the understanding of the real effects of indeterminate margins on prognosis.

Another point to consider is the possible different impact of indeterminate margins according to the extent of

\section{Reference} 2030-40.e4.

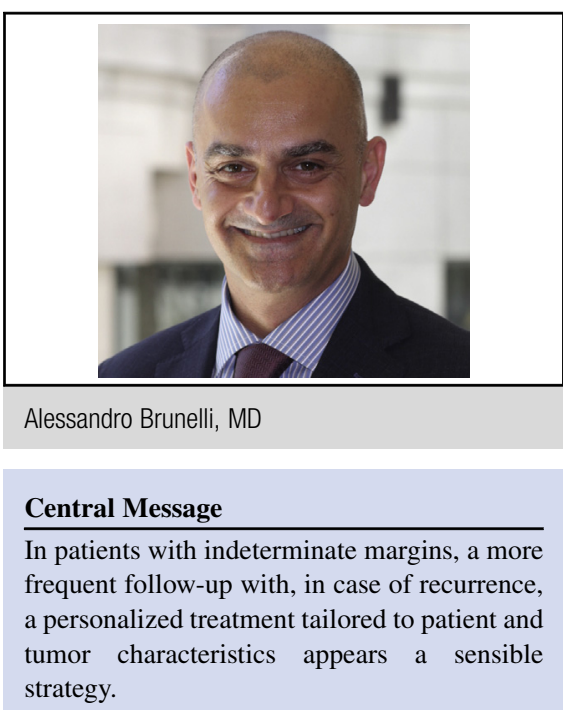

See Article page 2030.

operation. Indeterminate margins after pneumonectomies are related mostly to residual disease in the bronchial stump, whereas in lesser resections, the indeterminate margins may pertain to the lung parenchymal staple line, the lymph nodes, or, again, the bronchial stump. These different patterns may have a different biologic behaviors and prognostic implications. To clarify this aspect in part, Raman and colleagues ${ }^{1}$ performed a subgroup analysis that excluded pneumonectomies, which confirmed the negative results of adjuvant therapy.

What are the implications of this study? Certainly, in future databases we need more detailed definitions of indeterminate resection margins. The International Association for the Study of Lung Cancer staging committee has appointed an R-factor subcommittee to work on this aspect. As far as management of indeterminate margins is concerned, the study of Raman and colleagues ${ }^{1}$ may have a tremendous effect on our daily practice. In fact, it suggests that the routine use of adjuvant treatment should be discouraged. A more sensible approach appears to be the institution of a more intense surveillance with, in case of documented recurrence, a personalized treatment tailored to individual patient characteristics and cancer biology.

1. Raman V, Jawitz OK, Yang CFJ, Voigt SL, Kim AW, Tong BC, et al. The impact of adjuvant therapy on survival in patients with indeterminate margins following surgery for non-small cell lung carcinoma. J Thorac Cardiovasc Surg. 2020;159: 